\title{
Ecoescleroterapia com microespuma em varizes tronculares primárias
}

\author{
M icrofoam ultrasound-guided sclerotherapy in primary trunk varicose veins
}

\author{
M arcondes Figueiredo ${ }^{1}$, Salustiano Pereira de Araújo ${ }^{2}$, Nilson Penha-Silva ${ }^{3}$
}

\begin{abstract}
Resumo
O bjetivo: Avaliar o tratamento de varizes tronculares primárias por ecoescleroterapia com microespuma.

M étodos: A amostra foi constituída de 25 membros de seis pacientes do sexo masculino e 19 do sexo feminino, com incompetência das veias safenas magna (21 membros) ou parva (quatro membros). Eles foram avaliados de acordo com a classificação clínica, etiológica, anatômica e fisiopatológica (CEAP) e classificados nos graus $\mathrm{C}_{3}$ (10 membros), $C_{4}$ (seis membros), $C_{5}$ (cinco membros) e $C_{6}$ (quatro membros). A microespuma ( $5 \mathrm{ml}$ ), preparada pela mistura de $1 \mathrm{ml}$ de polidocanol a 3\% e $5 \mathrm{ml}$ de ar, era injetada na veia do paciente, em posição deTrendelenburg, com monitoração por ultra-sonografia com D oppler colorido. 0 s membros eram enfaixados com atadurainelástica por 3 dias; depois disso, os pacientes usavam meias elásticas, 30-40 mmH g, 3/4 ou 7/8, durante 3 meses. A eficiência do tratamento foi avaliada pelo escore clínico da classificação CEAP para dor, edema e claudicação e pelas alterações ultra-sonográficas 12 meses depois. 0 s escores clínicos antes e depois do tratamento foram comparados pelo teste de Wilcoxon.

Resultados: H ouve diminuição significante nos escores clínicos $(P<0,05)$ e sucesso terapêutico, com oclusão total e recanalização parcial sem refluxo, em $84 \%$ dos casos. D o total, $16 \%$ dos casos apresentaram recanalização parcial, com refluxo, ou recanalização completa.
\end{abstract}

C onclusão: A ecoescleroterapia, método simples, de baixo custo, que dispensa internação e anestesia, mostrou ser promissora no tratamento de varizes tronculares primárias.

Palavras-chave: Escleroterapia, varizes, polidocanol, ultrasonografia por D oppler colorido.

\footnotetext{
1. Angiologista e cirurgião vascular. Mestre em Ciências da Saúde, Universidade Federal de Uberlândia (UFU), Uberlândia, MG. Doutorando, Curso de Pós-Graduação em Angiologia e Cirurgia Vascular, Universidade Federal de São Paulo (UNIFESP), São Paulo, SP

2. Ultra-sonografista vascular, SBACV.

3. Professor titular, Instituto de Genética e Bioquímica, UFU, Uberlândia, MG. Doutor em Bioquímica, UFU, Uberlândia, MG. Pós-doutor em Bioquímica, Southern Illinois University (SIU), Carbondale, IL, EUA, e University of Texas Medical Branch, Galveston, TX, EUA.

Pesquisa desenvolvida no Hospital Santa Catarina, em Uberlândia, MG.
}

Artigo submetido em 25.07.06, aceito em 10.09.06.

J Vasc Bras 2006;5(3):177-83.

Copyright $\odot 2006$ by Sociedade Brasileira de Angiologia e de Cirurgia Vascular.

\begin{abstract}
O bjective: To evaluate the treatment of primary trunk varicose veins by microfoam ultrasound-guided sclerotherapy.

M ethods: The sample was composed of 25 members of six male and 19 female patients, with incompetence of great saphenous vein (21) and short saphenous vein (4). Patients were assessed according to the clinical, etiologic, anatomical and pathophysiological (CEAP) classification and divided into degrees $C_{3}(10), C_{4}(6), C_{5}(5)$ and $C_{6}$ (4). With the patients in the Trendelenburg position, microfoam $(5 \mathrm{ml})$, prepared by mixture of $1 \mathrm{ml}$ polidocanol at $3 \%$ and $5 \mathrm{ml}$ of air, was injected in their vein and monitored by color-flow D oppler ultrasonography. The members were then swaddled with inelastic bandage for 3 days; afterward, the patients used $30-40 \mathrm{mmH}$ g, 3/4 or $7 / 8$, elastic socks, during 3 months. Treatment efficiency was evaluated based on the CEAP classification for pain, edema and claudication, and on color-flow D oppler ultrasonographic changes 12 monthslater. Clinical scores, before and after treatment, were compared using the Wilcoxon test.
\end{abstract}

Results: There was a significant decrease in the clinical scores $(p<0.05)$. T herapeutic success was achieved, with total occlusion and partial recanalization without reflux in $84 \%$ of the cases. Theremaining $16 \%$ of the cases presented partial recanalization with reflux or complete recanalization.

Conclusion: Ultrasound-guided sclerotherapy, which is an easy and low-cost method that does not require hospitalization and anesthesia, proved to be promising for the treatment of primary trunk varicose veins.

Key words: Sclerotherapy, varicose veins, polidocanol, color-flow D oppler ultrasonography.

\section{Introdução}

As varizes são caracterizadas por tortuosidades, alongamentos e aumento no diâmetro das veias dos membros inferiores. Elas apresentam uma prevalência de aproximadamente $37,9 \%$ na população geral, sendo encontradasem $30 \%$ doshomenseem $45 \%$ das mulheres ${ }^{1}$. 0 tratamento de escolha das varizes tronculares primárias pelos cirurgiões vasculares brasileiros é a safenectomia total ou parcial, com ou sem a retirada de colaterais, embora sua taxa de recorrência em 5 anos esteja entre 20 e $80 \% 2$. 
A escleroterapia com a introdução de bolhas de ar na veia, para retirar o sangue emelhorar a ação terapêutica do esclerosante no endotélio, foi descrita primeiro por 0 rbach $^{3}$ como alternativa para o tratamento de varizes. Entretanto, as incertezas determinadas pelas dimensões das bolhas de ar e sua distribuição na circulação do paciente constituíram, por muito tempo, fatores limitantes para sua utilização.

A constatação da capacidade de al guns esclerosantes de produzir microespumas com potente ação terapêutica levou a um crescimento do interesse pela escleroterapia e sua utilização no tratamento de varizes de grandes vasos. A associação da escleroterapia com a ultra-sonografia com D oppler colorido levou ao advento da ecoescleroterapia ${ }^{4-9}$.

A ecoescleroterapia com microespuma apresenta várias vantagens em relação à escleroterapia líquida: 1) a microespuma permite o preenchimento de grandes espaços dentro das veias, a partir de volumes bem pequenos do líquido esclerosante misturado com ar; 2) a microespuma promoveo deslocamento do sangue no interior da veia varicosa durantea suainjeção, permitindo um contato mais longo e intensivo com o endotélio do vaso; 3) a injeção da microespuma promove um vasoespasmo na veia; 4) a microespuma apresenta uma perfeita ecovisibilidade por ultra-sonografia com D oppler colorido, o quetorna o método bastante seguro; 5) a microbolha apresenta uma estabilidade deno mínimo 30 minutos, garantindo, assim, uma elevada eficácia terapêutica.

Este trabalho relata resultados preliminares de um estudo prospectivo sobre o uso de ecoescleroterapia de varizes tronculares primárias à base de polidocanol, esclerosante com ação detergente, capaz de formar microespumas, e que apresenta baixa toxicidade e rara anafilaxia.

\section{M étodos}

0 presentetrabalho foi previamente aprovado pelo comitê institucional de ética do H ospital Santa C atarina, em U berlândia (M G).

\section{Amostragem}

O s pacientes com varizes tronculares que atendemos em clínica privada, de julho de 2003 a julho de 2004, foram avaliados de acordo com a classificação clínica, etiológica, anatômica efisiopatológica (CEAP). Aqueles pacientes que apresentavam antecedentes de trombose venosa profunda, recente ou tardia, trombofilia, gestação, neoplasia einsuficiência arterial periférica (índice tornozelo-braço $<0,8$ ) foram excluídos do estudo. 0 sdemais pacientes receberam uma explicação detalhada sobre o tratamento proposto, e aqueles que concordaram assinaram termo de consentimento livre e esclarecido. Foram tratados 25 membros de seis pacientes do sexo masculino ede 19 do sexo feminino, com idademédia de 47 anos. D os 25 membrostratados, 21 estavam com incompetência de veia safena magna, e quatro, com comprometimento de veia safena parva. Elesforam classificadosnosgraus $\mathrm{C}_{3}$ (10 membros), $\mathrm{C}_{4}$ (seis membros), $C_{5}$ (cinco membros) e $C_{6}$ (quatro membros) por exame clínico e ultra-sonografia com D oppler colorido.

Para preparação da microespuma de polidocanol, uma seringa de $3 \mathrm{ml}$, contendo $1 \mathrm{ml}$ de polidocanol a $3 \%$, eraconectadaaumaseringa de $5 \mathrm{ml}$, contendo $5 \mathrm{ml}$ de ar, por meio de uma torneira de infusão de 3 vias (Figura 1); após 20 movimentos na mistura do ar com o líquido, era obtida a microespuma, ou espuma densa, como ela é também designada ${ }^{10}$.

\section{Ecoescleroterapia}

Com o paciente na posição de Trendelenburg, a veia safena magna ou parva era visibilizada, Iongitudinalmente, a uma distância de $20-25 \mathrm{~cm}$ da junção safeno-femoral, por ultra-sonografia com D oppler colorido. U m volume de $5 \mathrm{ml}$ da microespuma era injeta-

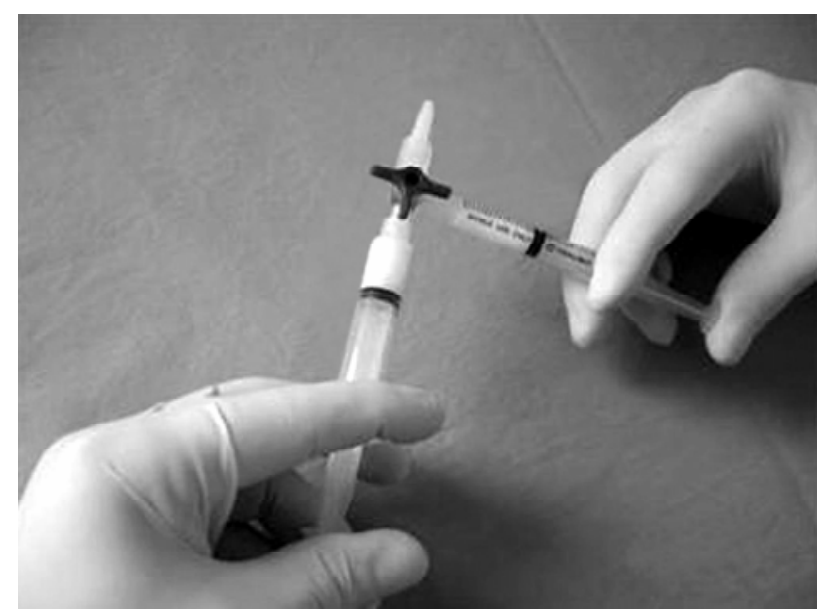

Figura 1 - Produção da microespuma em sistema constituído pelajunção de duas seringas com 3 e $5 \mathrm{ml}$ de capacidade, por meio de uma torneira de infusão de 3 vias 
do no interior da veia ou em suas colaterais, com o auxílio de uma agulha com dimensões de 0,7 versus 30 $\mathrm{mm}$, no terço médio da coxa, com compressão da junção safeno-femoral por 15 minutos, para evitar a progressão demicroespuma no sistema venoso profundo. A necessidade de novas sessões de ecoescleroterapia foi avaliada por ultra-sonografia após 1 e 6 meses da data da primeira sessão. 0 número de sessões utilizadas de microespuma foi de uma (seis pacientes), duas (14 pacientes) quatro (cinco pacientes), com uma média de 1,96 sessões por paciente. 0 membro era, então, enfaixado por 72 horas com atadura de baixa elasticidade. $\mathrm{N}$ os 3 meses seguintes, os pacientes usavam meia elástica, $30-40 \mathrm{mmH} \mathrm{g}$, modelo $3 / 4$ ou 7/8, e depois recebiam alta do procedimento, com restrição de exercício físico por 30 dias.

\section{M onitoração do tratamento}

A eficiência do tratamento foi monitorada com base nos escores para dor, edema eclaudicação, obtidos com a classificação CEAP ${ }^{11}$, antes edepois do procedimento, e nas ultra-sonografias com D oppler colorido, 12 meses após o tratamento, com definição de quatro categorias de resultados: 1) oclusão total; 2) recanalização parcial sem refluxo; 3) recanalização parcial com refluxo; e 4) recanalização completa ${ }^{12}$.

\section{Análise estatística dos resultados}

O s escores clínicos depois do tratamento foram comparados com os escores antes do tratamento, pelo teste de Wilcoxon, com valores de $\mathrm{P}<0,05$ indicando diferenças estatisticamente significantes entre eles.

\section{Resultados}

A T abela 1 apresenta os escores clínicos obtidos para aval iação dedor, edema eclaudicação, com basena classificação CEAP.

A T abela 2 apresenta os resultados finais, após 360 dias do tratamento, obtidos por ultra-sonografia com D oppler colorido. D os membros tratados (21 membros), 84\% apresentaram oclusão total ou recanalização parcial sem refluxo, situações que caracterizam sucesso da intervenção terapêutica ${ }^{12}$. A penas $16 \%$ dos casos (quatro membros) apresentaram recanalização parcial com refluxo ou recanalização completa. A Figura 2 apresenta uma situação visualizada por ultra-sonografia, em que houve oclusão total da junção safeno-femoral.

As complicações mais comuns do tratamento foram varicoflebite (Figura $3 \mathrm{~A}$ ) e hipercromia no trajeto das varicosidades. As varicoflebites foram tratadas por

T abela 1 - Escores clínicos (média \pm desvio padrão) para dor, edema e claudicação, antes e depois do tratamento, de acordo com a classificação clínica CEAP

\begin{tabular}{|c|c|c|c|c|c|}
\hline \multirow[t]{2}{*}{ CEAP } & \multirow[t]{2}{*}{$n$} & \multirow[t]{2}{*}{ Critérios } & \multicolumn{2}{|c|}{ Escores } & \multirow[t]{2}{*}{$\mathbf{P}$} \\
\hline & & & Antes & D epois & \\
\hline 3 & 10 & $\begin{array}{c}\text { dor } \\
\text { edema } \\
\text { claudicação }\end{array}$ & $\begin{array}{l}1,10 \pm 0,74 \\
0,80 \pm 0,79 \\
0,20 \pm 0,42\end{array}$ & $\begin{array}{l}0,40 \pm 0,52 \\
0,22 \pm 0,44 \\
0,00 \pm 0,00\end{array}$ & $\begin{array}{c}0,0117 * \\
0,0277 * \\
0,1797\end{array}$ \\
\hline 4 & 6 & $\begin{array}{c}\text { dor } \\
\text { edema } \\
\text { claudicação }\end{array}$ & $\begin{array}{l}1,50 \pm 0,55 \\
1,50 \pm 0,55 \\
1,00 \pm 0,63\end{array}$ & $\begin{array}{l}0,67 \pm 0,52 \\
0,33 \pm 0,52 \\
0,33 \pm 0,52\end{array}$ & $\begin{array}{c}0,0431 * \\
0,0277 * \\
0,0679\end{array}$ \\
\hline 5 & 5 & $\begin{array}{c}\text { dor } \\
\text { edema } \\
\text { claudicação }\end{array}$ & $\begin{array}{l}2,00 \pm 0,00 \\
2,00 \pm 0,00 \\
1,60 \pm 0,55\end{array}$ & $\begin{array}{l}0,80 \pm 0,45 \\
0,80 \pm 0,45 \\
0,40 \pm 0,55\end{array}$ & $\begin{array}{l}0,0431 * \\
0,0431 * \\
0,0431 *\end{array}$ \\
\hline 6 & 4 & $\begin{array}{c}\text { dor } \\
\text { edema } \\
\text { claudicação }\end{array}$ & $\begin{array}{l}2,00 \pm 0,00 \\
1,75 \pm 0,50 \\
1,50 \pm 0,58\end{array}$ & $\begin{array}{l}0,75 \pm 0,50 \\
0,50 \pm 0,58 \\
0,75 \pm 0,50\end{array}$ & $\begin{array}{l}0,0679 \\
0,0679 \\
0,1088\end{array}$ \\
\hline
\end{tabular}

CEAP = classificação clínica, etiológica, anatômica e fisiopatológica. 


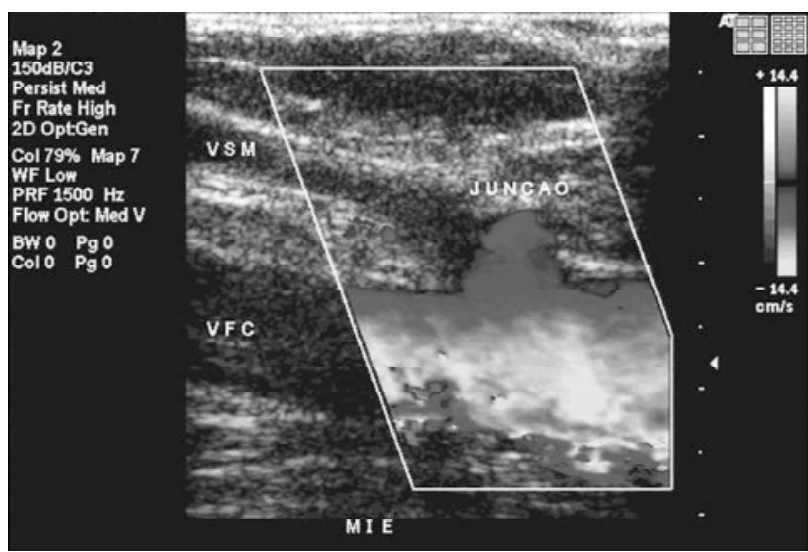

Figura 2 - M apeamento duplex mostrando uma junção safeno-femoral ocluída

drenagem aspirativa (Figura 3B) ou mecânica (Figura $3 C$ ), com bonsresultados (Figura 3D). Eventualmente, houve necessidade do uso de anal gésico e antiinflama- tório. Em nenhuma situação foi verificada trombose venosa profunda nos membros tratados, nem distúrbios visuais, dor torácica ou tosse, durante ou após os procedimentos da ecoescleroterapia.

As Figuras 4A e 4B apresentam uma paciente com varizes tronculares primárias antes (Figura 4A) edepois do tratamento (Figura 4B). 0 resultado estético foi satisfatório. Embora não tenha sido quantificada sistematicamente, a satisfação dos pacientes foi excelente.

\section{D iscussão}

A microespuma ideal é aquela que apresenta ecovisibilidade e uma ação esclerosante potente e longa ${ }^{6}$. 0 método que utilizamosna produção damicroespuma ${ }^{10}$ écapaz degerar microbol hasecovisíveis, com diâmetros entre 100 e $400 \mu \mathrm{m}$ e terapeuticamente ativas ${ }^{6}$. Como um detergente, o polidocanol é capaz de se solubilizar em água e de se misturar com os lipídeos que formam as membranas biológicas, promovendo sua ruptura e
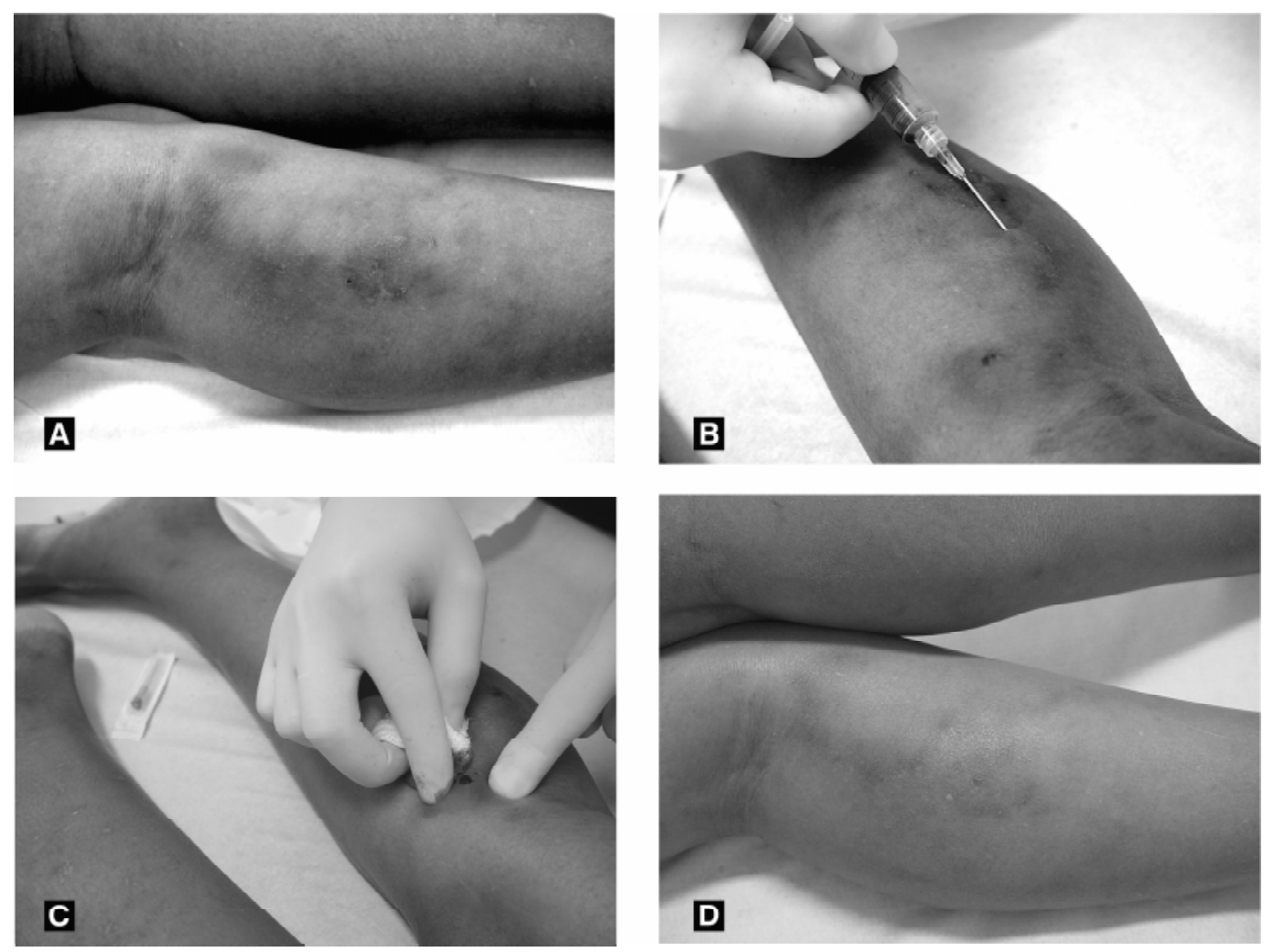

Figura 3 - A) V aricoflebite decorrente de ecoescleroterapia com microespuma. B) T ratamento de varicoflebite por drenagem aspirativa. C) Tratamento de varicoflebite por drenagem mecânica. D ) Resultado estético final 

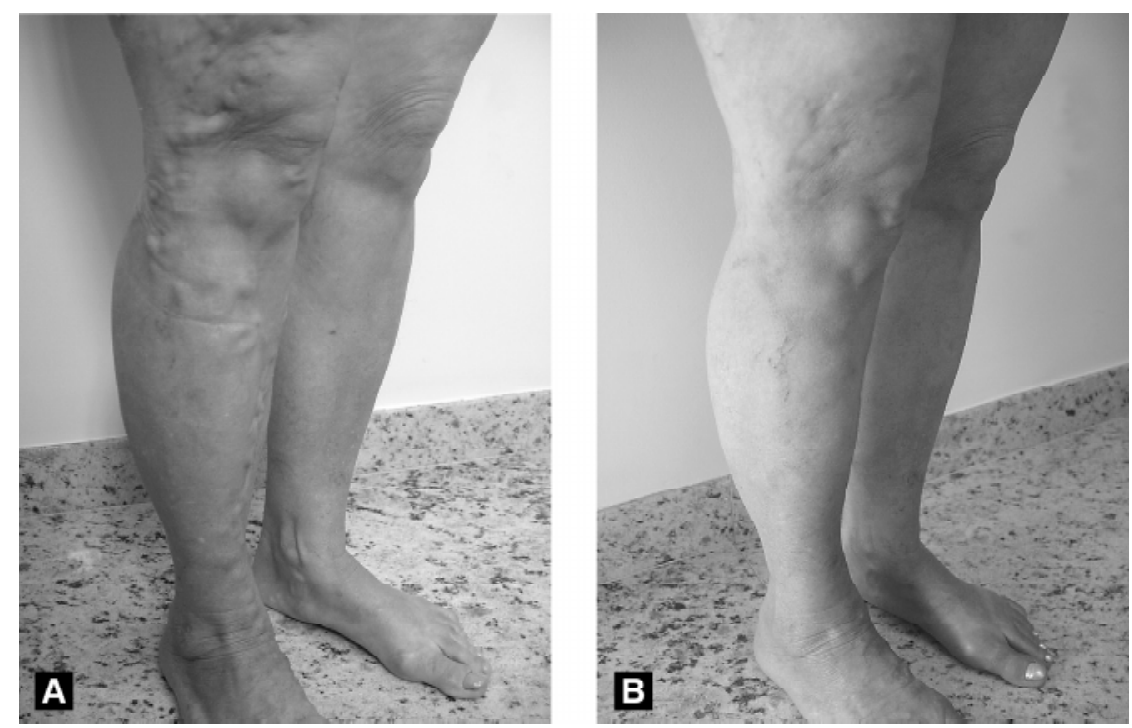

Figura 4 - A) Pacienteantes do tratamento por ecoescleroterapia com microespuma. B) Paciente depois do tratamento por ecoescleroterapia com microespuma

hiper-hidratação e morte da célula, com a formação de um macerado celular, queage como um trombo químico no interior do vaso.

A compressão pós-tratamento com microespuma, apesar denão ser unanimidadeno consenso europeu ${ }^{13}$, éapontadacomo necessária ${ }^{14}$, emboranão hajaconsenso sobre qual tipo de compressão deva ser utilizado. $\mathrm{N}$ este trabalho, foi inicialmente utilizada compressão inelástica, por permitir ao paciente permanecer de 3 a 5 dias com um único enfaixamento, uma vez que ele não compromete o fluxo sangüíneo. A póso quinto dia, com duração de até 3 meses, foi utilizada meia terapêutica para promover compressão elástica do vaso.

0 objetivo da escleroterapia não é a trombose do vaso, que está sujeito à recanalização, mas sim sua transformação em um cordão fibroso ${ }^{13}$.

A monitoração do membro do paciente por ultrasonografia com Doppler colorido após o tratamento permite a definição de quatro situações: 1) oclusão total; 2) recanalização parcial sem refluxo; 3) recanalização parcial com refluxo; e4) recanalização completa. 0 sucesso do tratamento é caracterizado pela ocorrência de oclusão total ou recanalização parcial sem refluxo, enquanto o insucesso é caracterizado pela recanalização parcial com refluxo ou recanalização completa ${ }^{12}$.
N este trabalho, a taxa de sucesso foi de $84 \%$, ao final de 1 ano depois do tratamento (T abela 2), o quede fato refletiu na melhora clínica significante dos pacientes, verificada pelo declínio dos escores clínicos dos sinais e sintomas dor, edema e claudicação ${ }^{11}$ naquele período (T abela 1). Em algumas situações, o declínio não foi significativo, certamente porque a amostra ainda não foi suficientemente grande. À medida que tenhamos mais pacientes para inserir nessa avaliação, deveremos ter uma idéia mais clara da eficiência do tratamento.

Ao final de 1 ano, a taxa de insucesso destetrabalho foi de $16 \%$ (quatro casos). A recanalização nem sempre resulta em recorrência deveiasvaricosas, masimplicana

Tabela 2 - Resultado da ultra-sonografia com D oppler colorido 12 meses após o tratamento

\begin{tabular}{lcc}
\hline Situação & N úmero & $\%$ \\
\hline O clusão & 12 & 48 \\
Recanalização parcial sem refluxo & 9 & 36 \\
Recanalização parcial com refluxo & 2 & 8 \\
Recanalização total com refluxo & 2 & 8 \\
Total & 25 & 100 \\
\hline
\end{tabular}


necessidade de acompanhamento regular. A taxa de recorrência reportada na literatura, em estudos com acompanhamento dos pacientes por 3 anos após o tratamento por ultra-sonografia com D oppler colorido, éde 20 a $33 \%^{11}$. Como nosso acompanhamento foi de apenas 1 ano, é difícil estabelecer uma comparação. Com certeza, é preciso que tenhamos também um acompanhamento por um maior período de tempo, cerca de 3 a 5 anos, para poder avaliar definitivamente a eficiência dessa terapêutica.

Embora tenhamos tido $84 \%$ de oclusão e recanalização parcial sem refluxo ao final de 1 ano de acompanhamento, a taxa de recanalização (parcial sem refluxo, parcial com refluxo e total com refluxo) representou $52 \%$ da amostra (T abela 2). Somente a extensão desse acompanhamento permitirá avaliar o prognóstico das recanalizações quanto ao refluxo, o que é de suma importância neste trabalho.

As complicações mais comuns decorrentes do tratamento descrito em nosso trabalho foram varicoflebite ehipercromia no traj eto das varicosidades. O s casos de varicoflebite foram tratados com sucesso, usando drenagem manual ou aspirativa (Figura 2). A hiperpigmentação depelemel horou espontaneamente ao longo de 12 meses, com ou sem uso de medicação tópica.

$\mathrm{N}$ um estudo feito pelo $\mathrm{N}$ ational Institutefor $\mathrm{Clini-}$ cal Excellence ${ }^{15}$, varicoflebite também foi a complicação maiscomum, seguida por trombosevenosa profunda (TVP), necrose de pele e distúrbio visual, embora este tenha sido também descrito como a principal complicação do tratamento, porém em estudo onde se utilizou $10 \mathrm{ml}$ demicroespuma por sessão ${ }^{16}$, diferentemente deste trabalho, onde utilizamos um volume médio de $5 \mathrm{ml}$ de microespuma por sessão.

0 risco deT V P decorrentedo tratamento devarizes tronculares primárias por ecoescleroterapia não parece ser diferente do risco decorrente do procedimento cirúrgico, uma vez que a incidência estimada de TVP é de $3 / 1.000$ sessões de microespuma ${ }^{16}$ e de 2-5/1.000 cirurgias de varizes ${ }^{17}$.

A ocorrência de complicações respiratórias em de corrência da ecoescleroterapia com microespuma não foi detectada ${ }^{18}$. A avaliação pulmonar por cintilografia, após a perfusão venosa de $10 \mathrm{ml}$ de microespuma, não detectou alteração alguma ${ }^{19}$. Em nosso trabalho, nós também não detectamos nenhuma complicação respiratória, embora a nossa amostra seja ainda pequena, com 47 perfusões de microespuma nos 25 membros tratados.

A pós ecoescleroterapia com $20 \mathrm{ml}$ de microespuma de polidocanol na veia safena interna, foi descrita a ocorrênciadeum caso deacidentevascular cerebral, que foi atribuído a um forame ovale posteriormente diagnosticado no paciente ${ }^{20}$. Embora o volume demicroespuma usado naquele trabalho tenha sido quatro vezes maior queo volumequeutilizamos $(5 \mathrm{ml})$, éimportante destacar que o diagnóstico de forame ovale deve ser usado como critério de exclusão do paciente para tratamento com microespuma.

\section{Conclusão}

Resultados preliminares de ecoescleroterapia com microespumas do esclerosante polidocanol, no tratamento de varizes tronculares primárias, mostraram um sucesso terapêutico de $84 \%$, com 16\% apenas de recanalização parcial com refluxo ou recanalização completa, e uma diminuição significante nos critérios dor, edema e claudicação, avaliados de acordo com a classificação CEAP. As complicações mais comuns foram varicoflebiteehipercromia. Como o método ésimples, de baixo custo edispensa internação, anestesia erepouso, características desejáveis tanto pelo paciente quanto pelo médico, ele deve se tornar uma boa opção para tratamento de varizes tronculares primárias.

\section{Referências}

1. M affei FH A. Varizes dos membros inferiores: epidemiologia, etiopatogenia e fisiopatologia. In: M affei FHA, Lastória S, Yoshida WB, Rollo HA, editores. Doenças vasculares periféricas. Rio de Janeiro: M edsi; 2001. p. 1499-510.

2. Kostas T, Ioannou CV, Touloupakis E, et al. Recurrent varicose veins after surgery: a new appraisal of a common and complex problem in vascular surgery. Eur J Vasc Endovasc Surg. 2004;27:275-82.

3. O rbach EJ. Sclerotherapy of varicose veins: utilization of intravenous air block. Am J Surg. 1944;362-6.

4. CabreraG arrido J, C abreraG arcia-O ImedoJ R. N uevo método de esclerosis en las varices tronculares. Patol Vasc. 1995;4: 55-73.

5. Cavezzi A, Frullini A, R icci S, T essari L. T reatment of varicose veins by foam sclerotherapy: two clinical series. Phlebology. 2002;17:13-8.

6. Frullini A. Foam sclerotherapy: a review. Phlebolymphology. 2003;40:125-9.

7. Breu FX, Guggenbichler S. European consensus meeting on foam sclerotherapy. April, 4-6, 2003, T egernsee, G ermany. D ermatol Surg. 2004;30:709-17. 
8. Partsch B. D ie Schaumverödung: eine Renaissance der Sklerotherapie. Phlebologie. 2004;33:30-6.

9. Rossi GG. Escleroterapia eco-guiada com microespuma. In: Thomaz JB, Belczak CEQ, editores. T ratado de flebologia e linfologia. Rio de Janeiro: Rubio; 2006. p. 189-94.

10. T essari $L, C$ avezzi $A$, Frullini A. Preliminary experience with a new sclerosing foam in the treatment of varicose veins. D ermatol Surg. 2001;27:58-60.

11. Nicolaides AN; Cardiovascular Disease Educational and Research Trust; European Society of Vascular Surgery; The International Angiology Scientific Activity Congress O rganization; International Union of Angiology; Union Internationale de Phlebologie at the Abbaye des Vaux de Cernay. Investigation of chronic venous insufficiency: a consensusstatement (France, M arch 5-9, 1997). Circulation. 2000;102:E126-63. Review.

12. Yamaki T, N ozaki M, I wasaka S. Comparative study of duplex-guided foam sclerotherapy and duplex-guided liquid sclerotherapy for the treatment of superficial venous insufficiency. D ermatol Surg. 2004;30:718-22.

13. Rabe $E$, Pannier-Fischer $F, G$ erlach $H$, et al. Guidelines for sclerotherapy of varicose veins (ICD 10: 183.0, I83.1, I83.2, and 183.9). D ermatol Surg. 2004:30:687-93.

14. Agus GB, Allegra C, Antignani PL, et al. Guidelines for the diagnosisand therapy of the vein and lymphatic disorders. Int Angiol. 2005;24:107-68.

15. N ational Institute for Clinical Excellence. Interventional proceduresoverview of ultrasound guided foam sclerotherapy for varicose veins. London, UK: N ICE. 2004.
16. Guex JJ. Foam sclerotherapy: an overview of use for primary venous insufficiency. Semin V asc Surg. 2005;18:25-9.

17. Critchley $G, H$ anda $A, M$ aw $A, H$ arvey $A, H$ arvey $M R$, C orbett CR. Complications of varicose vein surgery. Ann $R$ Coll Surg Engl. 1997;79:105-10.

18. Bountouroglou D, Geroulakos G. U Itrasound-guided foam sclerotherapy for the treatment of primary varicose veins. Phlebology. 2004;19:107-8.

19. Belcaro G, Cesarone MR, Di Renzo A, et al. Foamsclerotherapy, surgery, sclerotherapy, and combined treatment for varicose veins: a 10-year, prospective, randomized, controlled, trial (VED IC O trial). Angiology. 2003;54:307-15.

20. Forlee M V, Grouden M, M oore DJ, Shanik G. Stroke after varicose vein foam injection sclerotherapy. J Vasc Surg. 2006;43:162-4.

\section{Correspondência:}

$M$ arcondes Figueiredo

Rua $M$ arques Povoa, 88

CEP 38400-438 - U berlândia, M G

E-mail: drmarcondes@gmail.com 